\title{
Adenosine-stress cardiac magnetic resonance imaging in suspected coronary artery disease: a net cost analysis and reimbursement implications
}

\author{
Guenter Pilz • Pankaj A. Patel • Ulrich Fell • \\ Joseph A. Ladapo · John A. Rizzo • Hai Fang • \\ Candace Gunnarsson - Tobias Heer $\cdot$ Berthold Hoefling
}

Received: 13 April 2010/Accepted: 21 May 2010/Published online: 4 June 2010

(C) The Author(s) 2010. This article is published with open access at Springerlink.com

\begin{abstract}
The health and economic implications of new imaging technologies are increasingly relevant policy issues. Cardiac magnetic resonance imaging (CMR) is currently not or not sufficiently reimbursed in a number of countries including Germany, presumably because of a limited evidence base. It is unknown, however, whether it can be effectively used to facilitate medical decision-making and reduce costs by serving as a gatekeeper to invasive coronary angiography. We investigated whether the application of CMR in patients suspected of having coronary
\end{abstract}

G. Pilz $(\bowtie) \cdot$ T. Heer · B. Hoefling

Department of Cardiology, Clinic Agatharied, Academic Teaching Hospital, University of Munich, Norbert Kerkel Platz, 83734 Hausham, Germany

e-mail: pilz@khagatharied.de

P. A. Patel

GE Healthcare, Barrington, IL, USA

U. Fell

GE Healthcare, Munich, Germany

J. A. Ladapo

Harvard Medical School, Boston, MA, USA

J. A. Rizzo

Stony Brook University, Stony Brook, NY, USA

H. Fang

University of Colorado, Aurora, CO, USA

C. Gunnarsson

S2 Statistical Solutions, Inc, Cincinnati, OH, USA artery disease (CAD) reduces costs by averting referrals to cardiac catheterization. We used propensity score methods to match 218 patients from a CMR registry to a previously studied cohort in which CMR was demonstrated to reliably identify patients who were low-risk for major cardiac events. Covariates over which patients were matched included comorbidity profiles, demographics, CAD-related symptoms, and CAD risk as measured by Morise scores. We determined the proportion of patients for whom cardiac catheterization was deferred based upon CMR findings. We then calculated the economic effects of practice pattern changes using data on cardiac catheterization and CMR costs. CMR reduced the utilization of cardiac catheterization by $62.4 \%$. Based on estimated catheterization costs of $€ 619$, the utilization of CMR as a gatekeeper reduced perpatient costs by a mean of $€ 90$. Savings were realized until CMR costs exceeded $€ 386$. Cost savings were greatest for patients at low-risk for CAD, as measured by baseline Morise scores, but were present for all Morise subgroups with the exception of patients at the highest risk of CAD. CMR significantly reduces the utilization of cardiac catheterization in patients suspected of having CAD. Per-patient savings range from $€ 323$ in patients at lowest risk of CAD to $€ 58$ in patients at high-risk but not in the highest risk stratum. Because a negative CMR evaluation has high negative predictive value, its application as a gatekeeper to cardiac catheterization should be further explored as a treatment option. 
Keywords Cardiac magnetic resonance imaging Adenosine stress - Myocardial perfusion · Coronary artery disease $\cdot$ Net cost analysis

\section{Introduction}

Adenosine-stress cardiac magnetic resonance (CMR) is an increasingly utilized technology in the noninvasive evaluation of patients suspected of having coronary artery disease (CAD). Several studies have demonstrated that CMR accurately detects significant CAD and that normal adenosine stress CMR results have high negative predict value [1-5]. However, researchers have not evaluated the economic implications of employing the technology as a gatekeeper to more expensive and invasive procedures such as cardiac catheterization.

In 2008, approximately 852,000 cardiac catheterizations were performed in Germany, translating into over $€ 500$ million in reimbursement for that year alone [6]. Clinicians and policymakers have raised concerns about its optimal utilization, as $60-70 \%$ of cardiac catheterization procedures yield negative results, and the procedure exposes patients to morbidities, including bleeding, radiation exposure, and contrast nephropathy. The low yield of cardiac catheterization was recently demonstrated in a study of nearly 400,000 patients without known coronary artery disease referred for elective catheterization. Only approximately one-third of the population were found to have obstructive coronary atherosclerosis [7]. The cost-effectiveness of diagnostic cardiac technologies is also an increasingly relevant policy issue internationally [8-13]. This is further heightened by the predictive power of less expensive technologies such as CMR. With these concerns in mind, the setting of these concerns, we performed a cost-analysis to explore the economic implications of using CMR as a decision-making instrument and gatekeeper to cardiac catheterization in a German care setting.

\section{Patients and methods}

Study population

We constructed a registry of all consecutive patients referred to our center for CMR evaluation within a seven-month period in 2007 and 2008. For the current analysis, our study group consisted of patients with suspected CAD who were candidates for invasive coronary angiography but first underwent stress CMR for further risk stratification. We refer to this group as the "Physician Gatekeeper" cohort, or PG. Data were collected on comorbidities, demographics, CADrelated symptoms, and CAD risk as measured by Morise scores [14]. Patients were followed to determine whether they were subsequently referred to cardiac catheterization after undergoing CMR.

\section{Stress CMR exam}

We considered exclusion criteria prior to performance of CMR, including standard CMR contraindications such as the presence of an internal pacemaker or defibrillator, cerebral aneurysm clips, metal in the eye, and contraindications to adenosine including history of asthma or bronchospasm. All anti-anginal medication and caffeinated beverages were stopped at least $24 \mathrm{~h}$ prior to the CMR exam. Informed consent was obtained from all patients. The protocol was approved by the local ethics committee.

CMR studies were performed with a $1.5 \mathrm{~T}$ magnetic resonance system (Signa Excite ${ }^{\circledR}$, GE Healthcare Milwaukee, USA) using an 8-element phased array surface coil (cardiac coil, GE Healthcare). In patients with claustrophobia, mild sedation with midazolame was offered. Cardiac function was assessed at rest in 3 long (two-chamber, threechamber, and four-chamber) and contiguous short axes using steady-state free precession sequences.

After infusing adenosine at a constant rate of $140 \mu \mathrm{g} / \mathrm{kg}$ per minute for $3 \mathrm{~min}$ (Spectris MR injector, Medrad, Indianola, USA), first-pass kinetics of a gadolinium-based contrast agent (Gadodiamide, Omniscan $^{\text {TM }}$, GE Healthcare Buchler, Germany; $0.1 \mathrm{mmol} / \mathrm{kg}$ ) was measured during breath-hold in 4-5 contiguous short axis orientations at every heart beat with a hybrid gradient echo/echo-planar pulse sequence (echo time $1.2 \mathrm{~ms}$, flip angle $25^{\circ}$, slice thickness $8 \mathrm{~mm}$, field of view 32-34 × 24-25.5 cm, matrix $128 \times 96$ ). This method has been described previously $[5,15]$. Ten minutes after stress perfusion, a second perfusion in the same orientation and with the same settings was performed at rest. Ten minutes after this second bolus, late enhancement images were acquired using an inversion-recovery prepared gated 

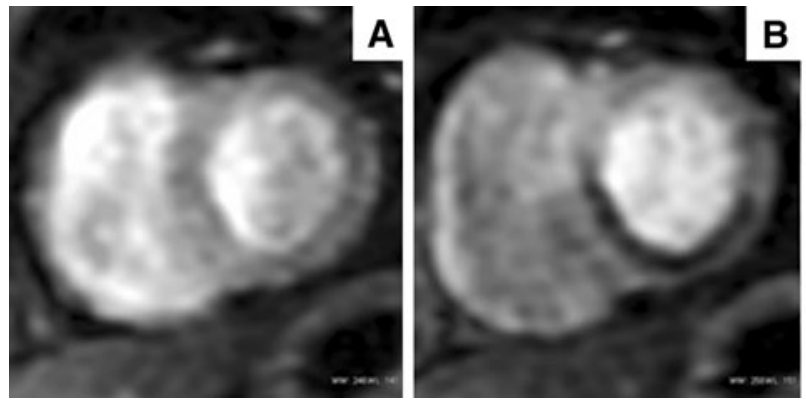

Fig. 1 Stress CMR - pathological findings. Short axis views of a normal first-pass perfusion under rest (a) but severe inferoseptal ischemia under adenosine-stress (b) and non-transmural

fast-gradient echo-pulse sequence (repetition time $6.7 \mathrm{~ms}$; echo time $3.3 \mathrm{~ms}$; flip $20^{\circ}$; inversion time individually adjusted; slice thickness $8 \mathrm{~mm}$; rectangular field of view 30 to $34 \mathrm{~cm}$; matrix $256 \times 160$ ). As planned in the perfusion study, 4-5 short axes and 3 long and contiguous short axes views using a 3D 20 slice sequence were acquired.

Two experienced investigators evaluated all CMR studies in consensus. Perfusion images were assessed visually. A perfusion deficit was regarded as relevant if it affected $>1 / 3$ of myocardial wall thickness in $\geq 2$ neighbouring segments, persisting $>5$ heart beats after maximal signal intensity in the cavity of the left ventricle (Fig. 1a and b). Late gadolinium enhancement (LGE) images were analysed visually and bright segments from subendocardial to epicardial were classified as fibrotic due to myocardial infarction (Fig. 1c and d).

Analysis and statistical model

The CMR registry included 605 patients. We eliminated those who met contraindications for CMR. We used propensity score methods [16] to match our study population (PG) to a previously-studied cohort in which CMR was demonstrated to reliably identify patients who were low-risk for major cardiac events (follow-up group, or FG) [5]. This control group consisted of 218 patients suspected of having CAD who were found to have a negative stress CMR. The negative predictive value of CMR in this cohort was 99.1\% and no patients experienced cardiac death, myocardial infarction, or other major adverse cardiac events (MACE) during a 12-month follow-up period. Through close matching, this control group served as
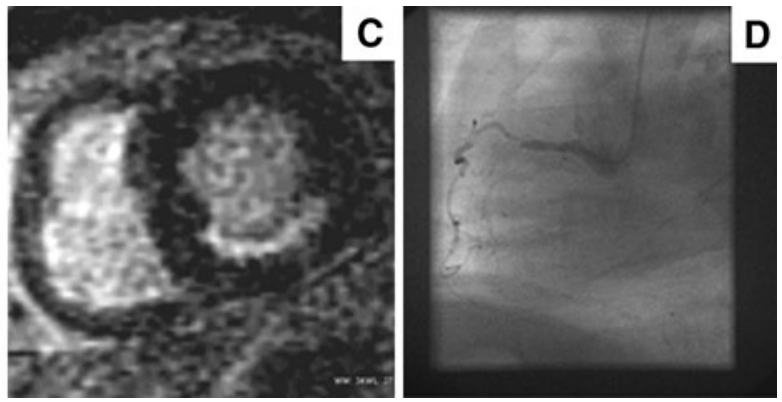

scar detection my means of LGE (c). Subsequent coronary angiography revealed chronic occlusion of the right coronary artery $(\mathbf{d})$

a proxy for expected outcomes in our study cohort, as long-term outcomes were otherwise unavailable.

Of the 605 patients in our original cohort, we eliminated 181 patients who had indications for CMR other than CAD assessment (Fig. 2) and 3 patients who did not complete a full CMR exam. This left a sample of 421 patients, compared to 218 patients in the FG cohort. A one-to-one propensity score match was used, resulting in a final matched sample of 218 PG patients. This approach was used to ensure a similar distribution of characteristics in the PG and FG cohorts. Matching criteria included: age, gender, body mass index, dyslipidemia, presence of diabetes, hypertension, and smoking status. The matched PG and FG cohorts did not differ significantly across major characteristics, including Morise score $(P=0.19)$ and other CAD risk factors $(P=0.47)$. The residual sample of 203 patients had similar results to the primary PG cohort but were not included in the analysis.

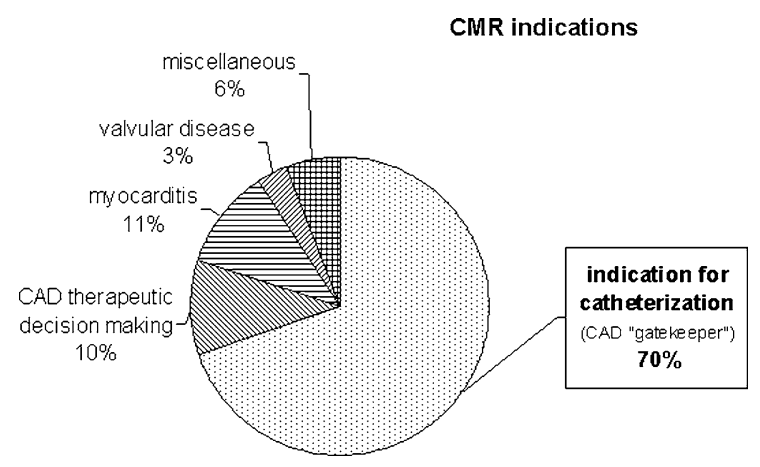

Fig. 2 Indications for CMR exams. Registry data on 605 consecutive patients 
Cost outcomes

We determined the portion of patients who were able to avoid cardiac catheterization based upon CMR findings. We then performed a cost analysis using data on the costs of cardiac catheterization and CMR and the portion of averted cardiac catheterizations. Our analysis was performed from the payor perspective using 2009 data in Euros.

We estimated cardiac catheterization costs using the difference in relative value units (RVUs) for a hospitalization in Germany with and without catheterization. We then multiplied this figure by $€ 2,800$, the average accepted multiplier cost for hospitalizations in Germany in 2009 [17]. This yielded a catheterization cost of $€ 619$. Please refer to the Sect. "Appendix" for further details.

Because CMR is not currently reimbursed in Germany, we estimated its cost using the ratio of CMR to catheterization cost from the Centers for Medicare \& Medicaid Services (CMS) in the United States [18]. We then applied this ratio to our catheterization cost to yield an estimated cost of CMR of $€ 296$.

We performed an overall cost analysis across all patients suspected of having CAD and sub-analyses for patients with different pretest probabilities of $\mathrm{CAD}$, as determined by Morise scores. The subanalyses included the following Morise score categories: 0-4 (very low risk); 5-8 (low risk); 9-12 (low intermediate risk); 13-15 (high intermediate risk); 16-18 (high risk); and $>18$ (very high risk). Sensitivity analyses were performed on the cardiac catheterization deterrent rates to evaluate the impact of this key input on model results.

\section{Results}

Descriptive statistics

We enrolled 602 of 605 consecutive patients (99.5\%) scheduled to undergo stress CMR during a 7 month enrollment period. Patients varied in specific indications for CMR referral but all "CAD gatekeeper" patients were thought to likely undergo catheterization otherwise (Fig. 2).

Clinical characteristics of the matched PG and FG cohorts are displayed in Table 1 . These groups did not differ significantly by Morise score, CAD-related symptoms, or other characteristics used in the propensity score algorithm.

\section{Catheterization avoidance rates}

Patients who had preserved myocardial viability, as demonstrated by the absence of LGE, and normal CMR findings with first-pass perfusion under both rest and adenosine-stress experienced significantly lower rates of subsequent cardiac catheterization (Fig. 3).

The portion of cardiac catheterizations averted as a result of CMR findings is shown in Table 2. Sixtytwo percent of patients evaluated with CMR did not undergo subsequent catheterization. This rate varied by Morise score: patients with lower Morise scores and lower pretest probability of CAD had higher catheterization avoidance rates, while the opposite was true for patients with higher pretest probabilities.

Net cost analysis

The results of the cost analysis are summarized in Table 3. CMR is associated with average cost savings of $€ 90$ per patient. These savings are inversely correlated with baseline Morise score, with patients with the lowest baseline risk of CAD realizing the highest savings (€323). Moreover, cost savings were realized for every Morise sub-group with the exception of the highest risk cohort (Morise score $>18$ ) cohort, where costs increased.

Breakeven analysis

We performed breakeven analyses by varying CMR cost and the portion of averted cardiac catheterizations to determine when CMR would be cost neutral. This analysis demonstrated that the utilization of CMR would continue to yield cost savings until its cost exceeded $€ 386$ or its associated catheterization diversion rates fell to $48 \%$ (Table 3 ).

Sensitivity analysis

The results of the sensitivity analysis are displayed in Table 4. We varied the portion of averted cardiac catheterizations, cost of cardiac catheterization, and cost of CMR by $20 \%$ in both directions. We also 
Table 1 Descriptive statistics

\begin{tabular}{|c|c|c|c|c|c|}
\hline \multirow[t]{3}{*}{ Variables } & \multicolumn{4}{|l|}{ Mean $^{\mathrm{a}}$} & \multirow[t]{3}{*}{$P$ value $^{\mathrm{b}}$} \\
\hline & \multicolumn{2}{|l|}{ Follow up } & \multicolumn{2}{|c|}{ Matched gatekeeper } & \\
\hline & $N=218$ & & $N=218$ & & \\
\hline Morise score & 13.85 & $(3.8)$ & 14.2 & (3.0) & 0.19 \\
\hline CAD-related clinical presentation & & & & & 0.45 \\
\hline Anginal symptoms & 69.7 & $\%$ & 64.7 & $\%$ & \\
\hline Pathological stress test & 3.7 & $\%$ & 5.5 & $\%$ & \\
\hline Arrhythmia/risk factors & 26.6 & $\%$ & 29.8 & $\%$ & \\
\hline Angina $\mathrm{CCS}^{\mathrm{c}}$ & 1.79 & $(0.59)$ & 1.77 & $(0.57)$ & 0.72 \\
\hline Age & 63.2 & $(12.9)$ & 62.9 & $(13.7)$ & 0.83 \\
\hline BMI & 26.8 & $(4.6)$ & 26.4 & $(4.7)$ & 0.47 \\
\hline Male & 56.0 & $\%$ & 55.1 & $\%$ & 0.85 \\
\hline Dyslipidemia $\uparrow \dagger \dagger$ & 36.7 & $\%$ & 33.9 & $\%$ & 0.55 \\
\hline Diabetes $\dagger \dagger$ & 8.7 & $\%$ & 9.6 & $\%$ & 0.74 \\
\hline Hypertension $\dagger$ & 68.4 & $\%$ & 68.8 & $\%$ & 0.92 \\
\hline Smoker & 35.3 & $\%$ & 34.4 & $\%$ & 0.84 \\
\hline
\end{tabular}

$\dagger$ Systolic blood pressure $>140 \mathrm{~mm} \mathrm{Hg}$ and/or diastolic blood pressure $>90 \mathrm{~mm} \mathrm{Hg}$

$\dagger \dagger$ Fasting plasma glucose of $>126 \mathrm{mg} / \mathrm{dl}$ or 2-h postload glucose of $>200 \mathrm{mg} / \mathrm{dl}$ or symptoms of diabetes mellitus and random plasma concentration of $>200 \mathrm{mg} / \mathrm{dl}$

$\dagger \dagger \dagger$ Known total cholesterol $>250 \mathrm{mg} / \mathrm{dl}$ or long-term treatment with a lipid-lowering agent

a Standard deviations are in parentheses for continuous variables

b Student's $t$ test for continuous variables and Chi-square test for binary variables

c in symptomatic patients
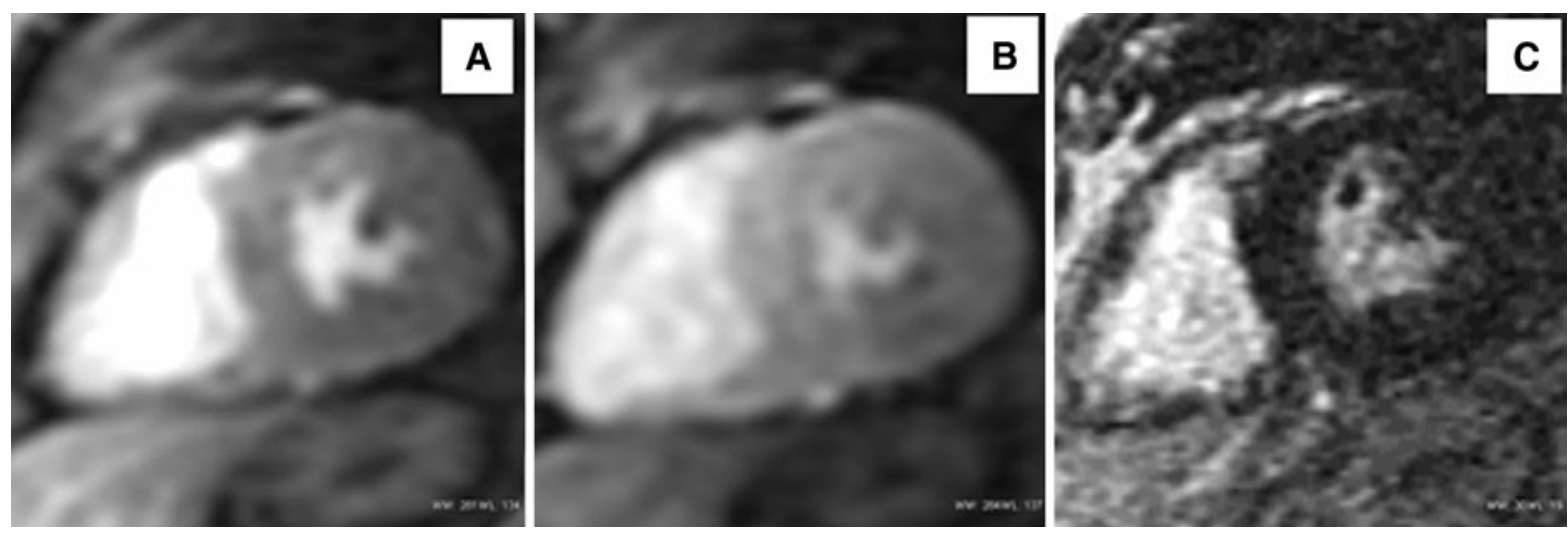

Fig. 3 Stress CMR - normal findings leading to catheterization avoidance in a patient with pre-test risk and clinical presentation comparable to the case in Fig. 1. Short axis views

report the results of the sensitivity analyses across Morise score subgroups. Cost savings remained greatest for patients with lower Morise scores but were present in most risk strata. However, for patients of a normal first-pass perfusion under both rest (a) and adenosine-stress (b) and absence of LGE (c)

with Morise scores greater than 16, using CMR as a gatekeeper would increase total costs if catheterization avoidance rates fell by $20 \%$ or the cost of catheterization or CMR increased by $20 \%$. 
Table 2 Cardiac catheterization avoidance rates

\begin{tabular}{lc}
\hline COHORT & Percent of CATHs avoided \\
\hline All & 62.4 \\
Morise: $0-4$ & 100.0 \\
Morise: $5-8$ & 85.7 \\
Morise: $9-12$ & 66.7 \\
Morise: $13-15$ & 64.1 \\
Morise: $16-18$ & 57.1 \\
Morise: $>18$ & 47.1 \\
\hline
\end{tabular}

Table 3 Net cost analysis*

\begin{tabular}{lc}
\hline COHORT & $\begin{array}{l}\text { Net cost savings } \\
\text { per patient (in €os) }\end{array}$ \\
\hline All & 90 \\
Morise: $0-4$ & 323 \\
Morise: $5-8$ & 235 \\
Morise: $9-12$ & 117 \\
Morise: $13-15$ & 101 \\
Morise: $16-18$ & 58 \\
Morise: $>18$ & -5
\end{tabular}

*Based on catheterization costs of $€ 619$ (see "Appendix" and Lit 14) and CMR costs of $€ 296$ (see Lit 15)

\section{Discussion}

Our study represents a preliminary investigation of the potential cost savings associated with utilizing CMR as a gatekeeper to invasive coronary angiography [19]. Cardiac catheterization is an expensive and invasive procedure, and major studies have demonstrated that, in select populations of CAD, it is not associated with an incremental survival benefit [20]. Moreover, the diagnostic yield of cardiac catheterization in confirming the presence of suspected CAD is poor [7, 21, 22]. Finally, because CMR evaluation has a high negative predictive value for disease-free survival, its application as a gatekeeper to cardiac catheterization should be further explored as a policy option.

We found that the utilization of CMR substantially reduced the need for cardiac catheterization in the diagnostic CAD work-up and was cost-saving even at reimbursement levels up to $€ 386$. Importantly, our population was primarily comprised of patients who
Table 4 Sensitivity analysis

\begin{tabular}{|c|c|c|}
\hline Model input & $+20 \%$ & $-20 \%$ \\
\hline \multicolumn{3}{|c|}{ CATH avoidance rate } \\
\hline All & 167 & 13 \\
\hline Morise: $0-4$ & 323 & 199 \\
\hline Morise: $5-8$ & 323 & 128 \\
\hline Morise: 9-12 & 199 & 34 \\
\hline Morise: $13-15$ & 180 & 22 \\
\hline Morise: $16-18$ & 128 & -16 \\
\hline Morise: $>18$ & 54 & -63 \\
\hline \multicolumn{3}{|l|}{ CATH cost } \\
\hline All & 167 & 13 \\
\hline Morise: $0-4$ & 447 & 199 \\
\hline Morise: $5-8$ & 341 & 128 \\
\hline Morise: 9-12 & 199 & 34 \\
\hline Morise: $13-15$ & 180 & 22 \\
\hline Morise: $16-18$ & 128 & -16 \\
\hline Morise: $>18$ & 54 & -63 \\
\hline \multicolumn{3}{|l|}{ CMR cost } \\
\hline All & 30 & 149 \\
\hline Morise: $0-4$ & 264 & 382 \\
\hline Morise: $5-8$ & 175 & 294 \\
\hline Morise: 9-12 & 57 & 176 \\
\hline Morise: $13-15$ & 42 & 160 \\
\hline Morise: $16-18$ & -2 & 117 \\
\hline Morise: $>18$ & -64 & 55 \\
\hline
\end{tabular}

would otherwise undergo cardiac catheterization, and cost savings were present across patients across a wide spectrum of pretest CAD risk. To our knowledge, this is the first documentation of the economic impact of CMR in a "real world" registry.

Beyond serving as an effective gatekeeper to cardiac catheterization, the utility of CMR as an instrument for risk stratifying patients with suspected CAD is also an important benefit. Researchers have demonstrated that a normal adenosine stress CMR has high predictive value. In a study of 461 patients with known or suspected CAD, Jahnke et al. found that three-year event-free survival was $99.2 \%$ for patients with a normal adenosine and dobutamine stress CMR [4]. We have also previously shown that a normal CMR is associated with a low MACE rate and favourable one-year prognosis in 218 patients with suspected CAD [5]. 
Our results are similar to other studies evaluating the utilization of non-invasive cardiac imaging technologies as gatekeepers to cardiac catheterization. In a recent paper on the German pilot phase of the EuroCMR registry, Bruder et al. reported that a substantial proportion of patients $(45 \%)$ did not undergo invasive angiography due to a normal CMR stress test [23]. The even higher overall catheter avoidance rate in our study may be due either to differences in patients' pre-test risk, to the exclusive use of adenosine stress in our center (higher negative predictive values than for dobutamine stress [1]) or to higher physicians' adherence rates to CMR results within a single-center setting. In our view, the latter aspect relying on the high negative predictive value of stress CMR in CAD evaluation deserves particular attention in future studies and registries. Clearly, physicians' compliance with the gatekeeper approach by waiving additional angiographies remains a prerequisite for cost-effectiveness. Høilund-Carlsen et al. found that single photon emission computed tomography (SPECT) reduced the need for catheterization by $50 \%$. Even in high-risk groups, substantial cost savings were attainable, and the risk of misidentifying patients with severe disease was low [24]. Shaw et al. performed a study in which SPECT was successfully used within a diagnostic pathway targeted towards selective resource utilization. They significantly reduced referrals to cardiac catheterization and attained cost savings by identifying low-risk patients who benefit from a more conservative care approach [11]. However, SPECT exposes patients to high doses of ionizing radiation, whereas CMR is a safer procedure $[25,26]$.

Our study has several limitations. We used matched populations to predict the risk of MACE in patients with negative CMR studies, as we did not have adequate follow-up in our study population. We also did not compare CMR to other noninvasive stress testing. As the sensitivity and specificity of noninvasive cardiac imaging modalities varies, the economic implication of their utilization as gatekeepers will also be affected. Our cost inputs were also associated with uncertainty, as CMR unit procedural costs and cardiac catheterization costs vary geographically [27]. Our study population may also not be representative of patients in other practice settings or with different demographic profiles. Finally, our analysis was performed from the payor perspective and may not be relevant to policymakers concerned with other perspectives such as the societal one.

\section{Conclusion}

CMR significantly reduces the utilization of cardiac catheterization in patients suspected of having CAD, and patients with normal CMR results have a low-risk of adverse cardiac events. We project that average per-patient costs would fall by $€ 90$ if CMR were routinely used as a gatekeeper to cardiac catheterization, and its application should be further explored by researchers and policymakers.

Acknowledgments The expert technical assistance of Eman Ali, RT, is gratefully acknowledged. GP discloses consultancy fees and research grants received from GE Healthcare. PAP and UF are employees of GE Healthcare. CG, JAR and JAL are paid consultants for GE Healthcare. The other authors have no competing interests to disclose.

Open Access This article is distributed under the terms of the Creative Commons Attribution Noncommercial License which permits any noncommercial use, distribution, and reproduction in any medium, provided the original author(s) and source are credited.

\section{Appendix}

Calculation of catheterization cost

Diagnostic catheterization costs within the German DRG system (reimbursement for hospitals/inpatient care) can be derived by comparing reimbursement for coronary arteriosclerosis without (DRG F66B, 1 day hospital stay) and with diagnostic catheterization (DRG F49F, 1 day hospital stay). Taking €2800 as an average 'base case value' for Germany this leads to catheterization costs of $€ 619$ for the year 2009.

The relative value for invasive cardiac diagnostic and 1 day hospital stay (DRG F49F) is 0.393 .

The relative value for DRG F66B, major diagnostic category for coronary arteriosclerosis without $\mathrm{CC}$, is 0.474 . For 1 day stay this value needs to be reduced by 0.302 which results in final relative value of 0.172 .

Subtracting 0.172 from 0,393 yields 0.221 , which is our measure of the change in relative value due to 
catheterization. Multiplying this difference, by the average base case value for Germany $€ 2,800$ ), results in $€ 618.80 \approx € 619$.

\section{References}

1. Nandalur KR, Dwamena BA, Choudhri AF, Nandalur MR, Carlos RC (2007) Diagnostic performance of stress cardiac magnetic resonance imaging in the detection of coronary artery disease - a meta-analysis. J Am Coll Cardiol 50:1343-1353

2. Schwitter J, Wacker CM, van Rossum AC, Lombardi M, Al-Saadi N, Ahlstrom H, Dill T, Larsson HBW, Flamm SD, Marquardt M, Johansson L (2008) MR-IMPACT: comparison of perfusion-cardiac magnetic resonance with single-photon emission computed tomography for the detection of coronary artery disease in a multicentre, multivendor, randomized trial. Eur Heart J 29:480489

3. Ingkanisorn WP, Kwong RY, Bohme NS, Geller NL, Rhoads KL, Dyke CK, Paterson DI, Syed MA, Aletras AH, Arai AE (2006) Prognosis of negative adenosine stress magnetic resonance in patients presenting to an emergency department with chest pain. J Am Coll Cardiol 47:14271432

4. Jahnke C, Nagel E, Gebker R, Kokocinski T, Kelle S, Manka R, Fleck E, Paetsch I (2007) Prognostic value of cardiac magnetic resonance stress tests: adenosine stress perfusion and dobutamine stress wall motion imaging. Circulation 115:1769-1776

5. Pilz G, Jeske A, Klos M, Ali E, Hoefling B, Scheck R, Bernhardt P (2008) Prognostic value of normal adenosinestress cardiac magnetic resonance imaging. Am J Cardiol 101:1408-1412

6. Bruckenberger E (2009) Herzbericht 2008 mit Transplantationschirurgie

7. Patel MR, Peterson ED, Dai D, Brennan JM, Redberg RF, Anderson HV, Brindis RG, Douglas PS (2010) Low diagnostic yield of elective coronary angiography. N Engl J Med 362:886-895

8. Garber AM, Solomon NA (1999) Cost effectiveness of alternative test strategies for the diagnosis of coronary artery disease. Ann Intern Med 130:719-728

9. Kuntz KM, Fleischmann KE, Hunink MG, Douglas PS (1999) Cost effectiveness of diagnostic strategies for patients with chest pain. Ann Intern Med 130:709-718

10. Rumberger JA, Behrenbeck T, Breen JF, Sheedy PF 2nd (1999) Coronary calcification by electron beam computed tomography and obstructive coronary artery disease: a model for costs and effectiveness of diagnosis as compared with conventional cardiac testing methods. J Am Coll Cardiol 33:453-462

11. Shaw LJ, Hachamovitch R, Berman DS, Marwick TH, Lauer MS, Heller GV, Iskandrian AE, Kesler KL, Travin MI, Lewin HC, Hendel RC, Borges-Neto S, Miller DD, For the Economics of Noninvasive Diagnosis (END) Multicenter Study Group (1999) The economic consequences of available diagnostic and prognostic strategies for the evaluation of stable angina patients: an observational assessment of the value of precatheterization ischemia. $\mathbf{J}$ Am Coll Cardiol 33:661-669

12. Dewey M, Hamm B (2007) Cost effectiveness of coronary angiography and calcium scoring using CT and stress MRI for diagnosis of coronary artery disease. Eur Radiol 17:1301-1309

13. Ladapo JA, Jaffer FA, Hoffmann U, Thomson CC, Bamberg F, Dec W, Cutler DM, Weinstein MC, Gazelle GS (2009) Clinical outcomes and cost-effectiveness of coronary computed tomography angiography in the evaluation of patients with chest pain. J Am Coll Cardiol 54:24092422

14. Morise A, Jalisi F (2003) Evaluation of pretest and exercise test scores to assess all-cause mortality in unselected patients presenting for exercise testing with symptoms of suspected coronary artery disease. J Am Coll Cardiol 42:842-850

15. Pilz G, Klos M, Ali E, Höfling B, Scheck R, Bernhardt $P$ (2008) Angiographic correlations of patients with small vessel disease diagnosed by adenosine-stress cardiac magnetic resonance imaging. J Cardiovasc Magn Reson $10: 8$

16. Rosenbaum PR, Rubin DB (1983) The central role of the propensity score in observational studies for causal effects. Biometrika 70:41-55

17. Source for DRG calculations (2009): http://www.g-drg. de/cms/index.php/inek_site_de/G-DRG-System_2009/Fall pauschalen-Katalog/Fallpauschalen-Katalog_2009

18. Source for US cost data: http://www.cms.hhs.gov/

19. Pilz G, Bernhardt P, Klos M, Ali E, Wild M, Höfling B (2006) Clinical implication of adenosine-stress cardiac magnetic resonance imaging as potential gatekeeper prior to invasive examination in patients with AHA/ACC class II indication for coronary angiography. Clin Res Cardiol 95:531-538

20. Boden WE, O'Rourke RA, Teo KK et al (2007) Optimal medical therapy with or without PCI for stable coronary disease. N Engl J Med 356:1503-1516

21. Madsen J, Bech J, Jørgensen E, Kastrup J, Kelbæk H, Saunamäki K (2002) Yield of 5, 536 diagnostic coronary arteriographies: results from a data registry. Cardiology 98:191-194

22. Zeymer U, Zahn R, Hochadel M, Bonzel T, Weber M, Gottwik M, Tebbe U, Senges J (2005) Indications, complications of invasive diagnostic procedures, percutaneous coronary interventions in the year 2003. Results of the quality control registry of the Arbeitsgemeinschaft Leitende Kardiologische Krankenhausärzte (ALKK). Z Kardiol 94:392-398

23. Bruder O, Schneider S, Nothnagel D, Dill T, Hombach V, Schulz-Menger J, Nagel E, Lombardi M, van Rossum AC, Wagner A, Schwitter J, Senges J, Sabin GV, Sechtem U, Mahrholdt H (2009) EuroCMR (European Cardiovascular Magnetic Resonance) Registry. Results of the German Pilot Phase. J Am Coll Cardiol 54:1457-1466

24. Høilund-Carlsen P, Johansen A, Christensen H, Vach W, Møldrup M, Bertram P, Veje A, Haghfelt T (2006) Potential impact of myocardial perfusion scintigraphy as gatekeeper for invasive examination and treatment in 
patients with stable angina pectoris: observational study without post-test referral bias. Eur Heart J 27:29-34

25. Fazel R, Krumholz HM, Wang Y, Ross JS, Chen J, Ting HT, Shah ND, Nasir K, Einstein AJ, Nallamothu BK (2009) Exposure to low-dose ionizing radiation from medical imaging procedures. N Engl J Med 361:849857
26. Lauer MS (2009) Elements of danger -The case of medical imaging. N Engl J Med 361:841-843

27. Pennell DJ, Sechtem UP, Higgins CB, Manning WJ, Pohost GM, Rademakers FE, van Rossum AC, Shaw LJ, Yucel EK (2004) Clinical indications for cardiovascular magnetic resonance (CMR): consensus panel report. Eur Heart J 25:1940-1965 